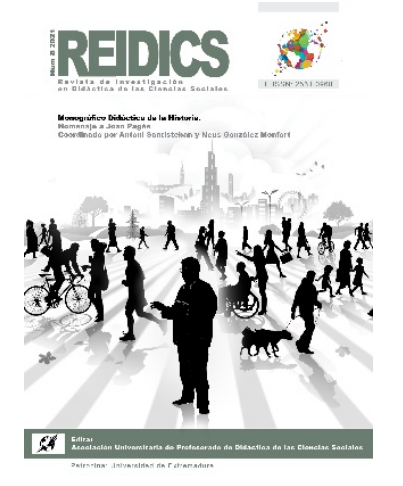

\title{
REIDICS
}

Revista de Investigación en

Núm. 8, 2021

Didáctica de las Ciencias

Sociales

Recibido 31 de enero de 2021

Aceptado 21 de marzo 2021

E-ISSN: 2531-0968

\section{Conocimiento histórico y formación ciudadana. Inclusión de otras narrativas históricas para una Nueva Historia Escolar}

\author{
Historical knowledge and citizenship education. Inclusion of \\ other historical narratives for a New School History
}

Gabriel Villalón-Gálvez

Universidad de Chile

Email: gabriel.villalon@uchile.cl

ORCID: http://orcid.org/0000-0003-0580-1870

DOI: https://doi.org/10.17398/2531-0968.08.89

\section{Resumen}

En este artículo presentamos una serie de ideas en relación con la Enseñanza de la Historia y la Ciudadanía. Primero presentamos las preguntas que dan origen a estas ideas sobre la Enseñanza de la Historia y la Ciudadanía y la construcción de una Historia Escolar que incorpore, fomente y desarrolle el abordaje de narrativas históricas vinculadas a otras y otros actores no tradicionales. A continuación, exponemos las razones porque planteamos la necesidad de una Nueva Historia Escolar y su relación con la construcción de la enseñanza de la ciudadanía. Por último, se exponen las ideas que sostienen la propuesta para la construcción de una Nueva Historia Escolar que incluya otras narrativas históricas, no tradicionales, y que fortalezca la formación ciudadana.

Palabras clave: enseñanza de la Historia; ciudadanía; educación; educación ciudadana; currículum.

\begin{abstract}
In this article we present a series of ideas in relation to the Teaching of History and Citizenship. First we present the questions that give rise to these ideas about the Teaching of History and Citizenship and the construction of a School History that incorporates, encourages and develops the approach of historical narratives linked to other non-traditional actors. Next, we state the reasons why we propose the need for a new School History and its relationship with the construction of the teaching of citizenship. Finally, we present the ideas that support the proposal for the construction of a School History that includes other non-traditional historical narratives and strengthens citizenship education.
\end{abstract}


Keywords: History education; citezenship; education; citezenship education; curriculum.

\section{A manera de introducción: Discusiones con Joan Pagès sobre una Enseñanza de los otros y las otras en la Historia Escolar}

Entre los años 2010 y 2014 mientras realizaba los estudios de doctorado iniciamos una discusión con Joan Pagès sobre qué historia se enseñaba en la escuela. Nuestra primera intención era dilucidar si el desarrollo historiográfico del siglo XX que habían abierto paso a lo que se denominó la Nueva Historia, la Historia Social y la Historia desde Abajo aparecía o no presente en el espacio de la Historia Escolar. En particular era de nuestro interés conocer si la historia de actores históricos no tradicionales relevada por estos desarrollos historiográficos había sido o no incorporada en la Historia Escolar. Según nuestra propuesta el que la Historia Escolar incorporará narrativas históricas sobre otras y otros actores históricos no tradicionales podría contribuir a la formación ciudadana, ya que esto colaboraría a que los y las estudiantes a través de la Historia se reconocieran como sujetos históricos y reconocieran su capacidad de agencia y desde ahí se desarrollaría una ciudadanía activa. Joan Pagès (2009) daba cuenta sobre la relación entre la Historia Escolar y la formación de ciudadanos y ciudadanas. Según Pagès (1994) las decisiones curriculares que se tomarán desde la enseñanza de la Historia podrían dar forma a distintos tipos de ciudadanos. Por esta razón Joan remarcaba la importancia de colocar atención a las propósitos y decisiones que conducían la enseñanza de la Historia y promovía la necesidad de que los y las docentes de Historia se preocuparán por visibilizar en clases la Historia de todas y todos aquellos que habían sido invisibles para la Historia tradicional y para la Historia en la escuela (Pagès, 1994; Pagès, 2003; Pagès, 2009; Villalón \& Pagès, 2013; Villalón \& Pagès, 2015). Pagès (2003 y 2009) planteaba la necesidad de que pensar y avanzar hacia una enseñanza de la Historia que tuviera como eje principal la formación democrática de los y las estudiantes y proponía que uno de los pilares de una enseñanza de la Historia que tuviera este propósito debía ser el preocuparse por dar a conocer y problematizar la Historia de aquellos y aquellos que tradicionalmente no estaban en los textos de la Historia Oficial.

De estas discusiones y reflexiones surgieron una serie de trabajos en conjunto en los que nuestro trabajo se concentró en analizar indagar sobre la presencia de actores y colectivos históricos no tradicionales en el currículum prescrito de Historia en Chile, España e Iberoamérica principalmente. (Pagès \& Villalón, 2013; Villalón \& Pagès, 2013; Villalón \& Pagès, 2015; Pagès, et al., 2017). A continuación, y como un homenaje a Joan Pagès presentó una propuesta que intenta recoger y sistematizar las ideas expuestas sobre esta temática en otros artículos junto a él y las ideas que nos quedaron por escribir sobre una Nueva Historia Escolar que tuviera como eje la incorporación de las narrativas históricas de actores históricos no tradicionales. 


\section{La Enseñanza de la Historia, sus usos y la representación de los grupos subalternos}

La preocupación por los relatos históricos que deben aprender las y los ciudadanos ha sido una preocupación permanente para los grupos de influencia y para el Estado. Zachos y Michailidou (2014) indican que en el contexto de la formación y consolidación de los Estados Nacionales en el siglo XIX la Educación Histórica tomó un rol relevante en cuanto se buscaba construir y fortalecer la idea de un pasado y una cultura común a través de la construcción de los mitos nacionales, además de la instalación de una serie de símbolos y prácticas rituales asociadas a la construcción de la Nación.

Considerando lo anterior, la comprensión de la Historia escolar que se instala desde el siglo XIX en la escuela, no puede ser entendida como un traspaso del conocimiento histórico disciplinar a la escuela, sino que debe ser comprendida como un área con fines propios. Cuesta (2000) indica que la educación histórica escolar significó la asignaturización del pasado que se instaló sobre un relato que tenía como foco validar al poder constituido por el Estado-Nación y las clases dominantes. Para esto el curso de Historia Escolar se organizó en torno al tratamiento de la Historia Universal y Nacional todo esto desarrollado bajo la influencia de las ideas del providencialismo, el progreso, el nacionalismo. En consecuencia, la Historia instalada como asignatura escolar desde el siglo XIX hasta la actualidad ha intentado responder a dos tipos de objetivos, por una parte, lo que se puede denominar objetivos románticos conativos relacionados con la construcción de los ideales nacionales. Y, por otra parte, los objetivos ilustrados-cognitivos referidos a la construcción desde el aprendizaje de la Historia de un razonamiento particular (Carretero, 2007; Toledo \& Gazmuri, 2009). Los primeros dominaron los currículos de Historia hasta los años ochenta para dar espacio a una mayor presencia del segundo tipo de contenidos en las disposiciones curriculares recientes, lo que sin embargo no se ha trasladado necesariamente a los recursos didácticos y las prácticas que se utilizan tradicionalmente en el aula escolar para enseñar la disciplina.

Los objetivos romántico conativos se han expresado en que la Historia Escolar se ha caracterizado por lo que la didáctica de la Historia ha calificado como un currículum tradicional o técnico (Pagès, 1994). Este currículum caracterizado por su tinte nacionalista, reproductivo y con un enfoque del aprendizaje centrado en la memorización concibe al pasado desde lo que Cuesta (2000), califica como una visión monumental y anticuaria. Lo que quiere decir una Historia escolar en la que se busca primero contemplar las grandes gestas del pasado que son impuestas por una memoria oficial que tiene su origen en el relato que imponen el Estado y los grupos dominantes. Y segundo una Historia escolar "que materializa el gusto por la conservación del pasado y un cierto sentido de apego a las raíces" (Cuesta, 2000, p. 27).

El dominio de la Historia tradicional ha significado que en la Historia Escolar se consoliden el desarrollo de narrativas históricas que se han caracterizado por su sentido tradicional, lo que se ha manifestado en cuestiones como la negación del conflicto y la historicidad de los grupos subalternos, por ejemplo como indican en su investigación Shear, et al. (2015) los discursos tradicionales de la enseñanza de la historia han silenciado las experiencias históricas de las minorías en Estados Unidos. Zachos y Michailidou (2014) han indicado que los relatos de los 
textos de estudio de Historia han tenido como características desarrollar un discurso histórico que niega la diferencia y que construye una representación del otro o la otra como peligroso o inferior, esta caracterización se ha utilizado tanto para caracterizar a todos y todas aquellas con los que los grupos dominantes tienen conflictos, ya sea a nivel interno o externo del país. De esta manera no ha sido habitual que en los relatos de la Historia Escolar figure un capítulo dedicado a los actores, a los protagonistas del devenir histórico o a los personajes y grupos invisibles. Por lo general, existe un predominio de la historia eurocéntrica y androcéntrica, de los relatos nacionales y de la tradición. La inclusión de actrices y actores históricos no tradicionales solamente es visualizada, cuando existen como un complemento al gran relato de la Historia Oficial, lo que en consecuencia significa que cualquier acción en contra de la hegemonía del proyecto estatal y de las clases dominante haya sido invisibilizado (Villalón \& Pagès, 2015; Pagès, et al., 2017).

\section{El desafío de una nueva Ciudadanía para la Enseñanza de la Historia}

La construcción de la ciudadanía a través de la escuela ha sido una constante preocupación del Estado. Durante el siglo XIX el proceso de construcción y consolidación del Estado-Nación tuvo entre sus principales acciones asegurar la formación de los ciudadanos de la nación. En este contexto la formación ciudadana estuvo identificada con la formación de una ciudadanía identificada con la nación (Pagès \& Santisteban, 2010). En el presente, los distintos fenómenos sociales y políticos que afectan al mundo, que se manifiestan en cuestiones como la desafección política, la individualización, la desigualdad y la cada vez más constante manifestación de discursos de tipo xenófobo y excluyentes, entre otros, han puesto la preocupación de una serie de organismos públicos internacionales y nacionales en los conocimientos políticos de las y los jóvenes, lo que ha dado fuerza a una serie de políticas que refuerzan la agenda de la formación ciudadana en las escuelas (Bolívar, 2007). Frente a las construcciones de una ciudadanía vinculada a los discursos nacionales hegemónicos y homogéneos en el siglo XXI se plantea el desarrollo de una formación ciudadana que reconfigure su perspectiva tradicional para colocar el acento en la construcción de un concepto de ciudadanía amplio, que haga visible la pluralidad de identidades, la diversidad y la alteridad como características de la democracia actual (Pagès \& Santisteban, 2010).

En la escuela la ciudadanía puede ser entendida como un concepto polisémico. Por una parte, puede estar asociado a la idea de civilidad o puede referirse a tener conductas respetuosas con las reglas de la vida en común; o corresponderse con la idea de educación cívica, que significa aprender cómo funcionan las instituciones; o puede vincularse a una perspectiva que coloca el foco en el estudio razonado y crítico de los problemas sociales y desde ahí conlleva una formación ciudadana con énfasis en el desarrollo de la participación ciudadana (Heimberg, 2010).

Esta última forma de concebir la formación ciudadana en la escuela es la que aparece como representativa de los proyectos de formación ciudadana como los que se han levantado en el mundo. Esta perspectiva ha sido denominada concepción máxima de la ciudadana y supone conocimientos sobre el sistema democrático y la institucionalidad política, pero, además el desarrollo de unas competencias por parte de los y las estudiantes (Cox, et.al., 2005), de unas habilidades, valores y disposiciones para que les permitan desenvolverse como ciudadanos 
plenos en una sociedad democrática y en la vida cívica en distintos niveles (Magendzo \& Gazmuri, 2018). El desarrollo de la ciudadanía en la escuela desde la perspectiva descrita significa un desafío en cuanto tiene como base sostenerse sobre procesos que tienen como foco el entender la democracia como un concepto inacabado, para lo cual sostiene que la base de la formación ciudadana está en que los y las estudiantes se enfrenten al desarrollo de la discusión de lo controversial y socialmente relevante en la escuela (Hess, 2009). Lo anterior, significa que la escuela debe enfrentarse a construirse como un espacio democrático, que debe ser capaz de abordar las distintas problemáticas que se desarrollan en el mundo real. Entre estos desafíos se encuentra el atender a la diversidad y convertirse en un espacio de inclusión (Bolívar, 2007; Heimberg, 2010; Magendzo \& Gazmuri, 2018).

En concordancia con lo anterior, planteamos que una enseñanza de la ciudadanía que se desarrolle sobre un enfoque máximo obliga a repensar la enseñanza de la Historia, ya que significa pasar de una enseñanza de la Historia desde un enfoque tradicional ligado a concepciones de ciudadanías mínimas y de tipo tradicional. Para avanzar a una enseñanza de la Historia que, a través del abordaje crítico del pasado, el reconocimiento de la multicausalidad y la multiperspectiva, así como de la agencia histórica de distintos actores y actrices históricas, la problematización del contenido histórico desde lo controversial otorgue la oportunidad a estudiantes de discutir el pasado y presente para poder proyectar el futuro. Desde esta perspectiva se apuesta por una Historia Escolar que permita acercar a los y las estudiantes a la experiencia de la democracia a partir del análisis del pasado.

\section{Ideas para la construcción de una historia escolar sobre las otras y los otros}

A continuación, presentamos un conjunto de ideas que creemos pueden servir de base para desarrollar una enseñanza de la Historia que permita el desarrollo de una ciudadanía máxima a partir de la visualización de la agencia histórica y política de otras y otros actores y colectivos históricos no tradicionales en la Historia escolar. Entendemos que una Historia Escolar que avance hacia la incorporación de otras narrativas puede convertirse en un medio para avanzar hacia la construcción de una enseñanza de la ciudadanía, que profundice en la democracia y la justicia social. La propuesta la organizamos en torno a tres ideas que se indican a continuación y se explicarán en los apartados que continúan:

1. La Historia como narrativa

2. La toma de decisiones sobre ideologías curriculares en la enseñanza de la Historia.

3. La formación del pensamiento histórico para visibilizar otras narrativas históricas y fortalecer la educación democrática.

\subsection{La Historia como narrativa}

Enseñar la Historia como una interpretación del pasado es clave si queremos profundizar en un acercamiento crítico de nuestros estudiantes al pasado, esto se encuentra directamente relacionado con la concepción de comprender la Historia como una narrativa y el rol que juegan 
esas narrativas en los significados que se hacen sobre el pasado, la comprensión del presente y proyección del futuro que harán nuestros estudiantes al desarrollar su conciencia histórica.

La Historia según White (2014) puede ser entendida como una narrativa, desde ahí la Historia es un relato que es una representación sobre el pasado, pero no es el pasado como tal. Los historiadores e historiadoras toman decisiones cuando interpretan el pasado, seleccionan fuentes, se hacen preguntas particulares las cuales por una parte responden a comprender cuestiones propias del pasado, pero también tienen una dimensión de interés que es propio del contexto histórico desde el que se investiga el pasado (Levstik \& Barton, 2015).

La Historia Escolar entendida como narrativa ha sido utilizada por los grupos de influencia y el Estado para ser una herramienta de construcción de discursos hegemónicos. Las narrativas históricas que han dominado la escuela se han caracterizado por invisibilizar cualquier relato que parezca contrahegemónico, esto quiere decir que la escuela ha obviado los conflictos en relación con el Estado y los distintos grupos sociales que componen la sociedad, como por ejemplo en el caso chileno la cultura Mapuche, las luchas de los pobladores, las mujeres y los movimientos sociales en general (Villalón \& Pagès, 2013; Villalón \& Pagès, 2015). En consecuencia, la Historia Escolar ha sido utilizada para la construcción de una conciencia histórica tradicional por parte de la ciudadanía.

El interés por el estudio del concepto de conciencia histórica ha respondido al entendimiento de la Historia como un fenómeno y no como un cuerpo de conocimientos predefinidos (Nordgren \& Johansson, 2015). Desde esta perspectiva adquiere relevancia el comprender los significados que construyen del pasado las personas a partir del conocimiento histórico. El concepto de conciencia histórica se ha considerado relevante para entender las funciones sociales de la enseñanza de la Historia. La conciencia histórica da cuenta del significado que dan las personas a la Historia a partir de la interacción simbiótica entre la interpretación del pasado, la comprensión del presente y la perspectiva del futuro (Nordgren \& Johansson, 2015). Desde esta perspectiva se entiende que la Enseñanza de la Historia tiene como principal finalidad que las personas le otorguen un sentido a la Historia, este sentido que se concreta desde el currículum, los instrumentos curriculares y las prácticas pedagógicas, responde a los intereses de los grupos de influencia que existen en cada sociedad (Seixas, 2017).

Rüsen (2008) plantea que la expresión de la conciencia histórica que poseen las personas se manifiesta a través de la competencia narrativa. La narrativa histórica puede ser entendida como la forma en que se da sentido al pasado, esta se desarrolla en torno a las siguientes tres competencias: experiencia, interpretación y orientación. La competencia histórica de la experiencia implica la capacidad de desarrollar la experiencia del pasado, esto quiere decir que las personas puedan observar el pasado con sus características temporales específicas y por lo tanto diferentes a las del presente. La competencia histórica de la interpretación se refiere a la capacidad de dar significado temporal a partir del establecimiento de las relaciones entre el pasado, el presente y el futuro. Este establecimiento de una comprensión del tiempo como un todo, se desarrolla en particular desde la temporalidad humana sobre la que las personas elaboran la interpretación a partir de la comprensión del pasado desde la relación con el presente y las expectativas sobre el futuro. Por último, la competencia histórica de la orientación implica la 
utilización que hacen del todo temporal (pasado, presente, futuro) las personas para orientar sus acciones y decisiones de vida. La competencia histórica de la orientación puede ser entendida como la articulación entre la identidad humana y el conocimiento histórico (Rüsen, 2008). Las narrativas históricas que se construyen por las personas y la sociedad articulan según Rüsen cuatro tipos de conciencia histórica: tradicional, ejemplar, crítica y genética.

Las narrativas y conciencia histórica que las personas poseen no es resultado exclusivo de los relatos históricos que se han aprendido y adquirido en la escuela, sin embargo, se debe reconocer que los discursos históricos que se representan desde los espacios educativos juegan un rol relevante en la comprensión del pasado, el presente y el futuro que desarrollan las personas. En consecuencia, las características que poseen las representaciones que se proyectan en la educación histórica escolar sobre los grupos históricos no tradicionales juegan un papel relevante sobre cómo serán los relatos sociales sobre el pasado, presente y futuro que construyan los y las jóvenes y el tipo de ciudadanía que se proyectará.

\subsection{La toma de decisiones sobre ideologías curriculares en la enseñanza de la Historia}

Una enseñanza de la Historia que considere el avance hacia una educación democrática a través de la incorporación de otras narrativas históricas requiere que los y las docentes revisen críticamente sus prácticas curriculares y tomen atención de los objetivos, contenidos, metodologías, estrategias y evaluaciones atendiendo que las decisiones que se toman sobre estos aspectos transmiten de manera explícita e implícita un discurso sobre la sociedad, un marco ideológico como ha sido definido desde la teoría curricular (Schiro, 2007; Eisner,1985; Carr, 1998). Por lo tanto, se hace necesario, para el logro del cambio de las prácticas educativas en Historia, que los docentes trabajen en la formación de saberes que permitan fortalecer las decisiones que se toman al momento de planificar y ejercer la enseñanza en búsqueda de que los estudiantes adquieran los aprendizajes para poder pensar históricamente y desarrollar una ciudadanía amplia. Esto exige primero que los docentes tengan conocimiento y tomen posición sobre el marco ideológico curricular sobre el que se desarrollan sus prácticas.

Las ideologías curriculares en Historia las podemos agrupar estas en tres perspectivas: la enseñanza de la Historia para la reproducción social; la enseñanza de la Historia cognitivista con énfasis en la formación del razonamiento de la disciplina y la enseñanza de la Historia con foco en la formación ciudadana (Pagès, 1994; Evans, 1990; Carretero, 2007; Gazmuri, 2017; Levesque \& Clark, 2018).

La enseñanza de la Historia para la reproducción social tiene como propósito el mantenimiento del status quo. En sus inicios estuvo vinculada a la construcción del Estado para luego desarrollarse con el objetivo de transmitir una narrativa que permitiera mantener y validar el proyecto social dominante. Esta perspectiva se ha calificado como un currículum tradicional o técnico de la Historia caracterizado por su tinte nacionalista, reproductivo y con un enfoque del aprendizaje centrado en la memorización (Pagès, 1994). Los objetivos reproductivos de esta ideología se expresan en una selección de contenidos que apuntan a que los y las estudiantes conozcan el pasado para así valorar las gestas del pasado y reconocer a los personajes que han 
guiado el avance continuo de la sociedad; el sentido reproductivo se expresa en que las metodologías utilizadas opten por estrategias que fortalecen lo transmisivo no existiendo espacio para el análisis crítico del pasado. Lo anterior es coherente con la evaluación en cuanto se utilizan instrumentos que permitan verificar si los y las estudiantes están reproduciendo el discurso enseñado (Pagès, 1994; Evans, 1990; Gazmuri, 2017). En cuanto a la ciudadanía promovida el sentido reproductivo de este currículum hace que lo promovido sea de carácter mínimo que coloca énfasis fundamentalmente en la participación representativa, los derechos políticos y la construcción de una identidad de tipo homogénea y asociada a la pertenencia a la nación (Kerr, 2015; Magendzo \& Gazmuri, 2018).

La enseñanza de la Historia cognitivista con énfasis en la formación del razonamiento de la disciplina tiene como objetivo que los y las estudiantes aprendan los conocimientos propios del análisis histórico (Carretero, 2007). Estos saberes propios del quehacer de la disciplina se han denominado Pensamiento Histórico el que puede ser definido como una serie de competencias intelectuales, copiadas del trabajo histórico, y por las que el estudiantado podrá construir, como este último, sus propios saberes históricos de manera más autónoma y no solamente consumir relatos ya construidos y ofrecidos por el profesor, aunque fueran de gran calidad (Pagès, 2009; Santisteban, 2010). Aprender a pensar históricamente significa desarrollar un proceso de alfabetización particular que serían los procedimientos propios de la ciencia histórica. Atendiendo al objetivo de que los y las estudiantes aprendan a pensar históricamente, los contenidos que se seleccionan se enfocan en aquellos que permiten aprender conocimiento de tipo procedimental vinculados a la Historia, como por ejemplo contextualización histórica, análisis de fuentes históricas o relaciones cambio y continuidad (Pagès, 2009; Santisteban, 2010; Levesque \& Clark, 2018; Van Boxtel \& Van Drie, 2018). Las metodologías colocan un acento importante en acercar a los y las estudiantes a trabajar con la investigación o el método histórico en el aula, del tipo de la metodología de trabajo que ha promovido el grupo de Sam Wineburg denominado "leyendo como historiadores" (2001). La evaluación desde esta perspectiva coloca su atención en cómo los y las estudiantes desarrollan el conocimiento procedimental histórico, por lo tanto, utiliza actividades evaluativas que permiten al estudiantado poner en práctica estos saberes respondiendo a problemas históricos. En cuanto a la ciudadanía no podemos establecer con claridad qué perspectiva pretende ser alentada. En principio algunos autores que han descrito el trabajo sobre el pensamiento histórico han señalado que aprender a pensar históricamente fortalecería las competencias ciudadanas hacia una lógica máxima, indicando que el desarrollo del pensamiento histórico es considerado como relevante porque es descrito como un instrumento intelectual de alto nivel, ya que puede permitir al futuro adulto encontrarse mejor en la constante decodificación de las noticias y las cuestiones sociales, económicas y políticas del presente y actuar así de manera más lúcida como ciudadano en la sociedad democrática a la que pertenece.

Una defensa clara de la premisa anterior ha sido el avance del trabajo del grupo de Stanford que teniendo como base su modelo para la formación del pensamiento histórico han generado metodologías de trabajo para favorecer en los estudiantes el análisis de la información disponible en redes sociales y páginas de internet (McGrew \& Byrne, 2020). Es claro que los objetivos y métodos de esta perspectiva la hacen más cercana a una ciudadanía máxima, en cuanto a que 
favorece por ejemplo la participación, la deliberación y el desarrollo del pensamiento crítico que puede relacionarse con ciudadanías más empoderadas, hay autores que critican esta perspectiva en cuanto indican se privilegia la formación de las competencias intelectuales asociadas al conocimiento histórico, más que a un abordaje que tenga como eje la formación ciudadana (Levesque \& Clark, 2018).

La tercera perspectiva surge de la crítica al enfoque cognitivista. Esta perspectiva plantea que la enseñanza de la Historia además de tener como propósito la formación del razonamiento histórico debe colocar la atención en construir una educación democrática (Barton \& Levstik, 2009). Esta perspectiva se encuentra asociada a planteamientos como los de Barton y Levstik que señalan que la enseñanza de la Historia debe tener como finalidad la búsqueda del bien común (2009). En esta línea la Historia escolar aparece como un espacio para la formación de razonamientos asociados a la elaboración de juicios, el trabajo sobre la deliberación y el activismo (Levesque \& Clark, 2018).

El aprendizaje de la Historia desde esta perspectiva es clave para la formación ciudadana porque permite a los y las estudiantes conectarse con las personas del pasado, lo que tiene como resultado que estas puedan sentirse parte de una comunidad más amplia. La Historia permite acercar a las personas al análisis crítico de la realidad a través de la comprensión causal de los fenómenos históricos, lo que amplía su visión para analizar las problemáticas del presente y les permite entenderlas desde una perspectiva histórica. La Enseñanza de la Historia permite a las personas poder enfrentarse a discutir cuestiones controversiales a partir del análisis y debate de las decisiones y acciones que han tomado las personas en el pasado. La Enseñanza de la Historia permite que las personas puedan aprender a buscar, indagar y analizar información para construir argumentaciones a partir del trabajo con las fuentes históricas, esto se entiende una competencia clave para ciudadanía contemporánea (Barton \& Levstik, 2009; Levesque \& Clark, 2018). Otros autores agregan a lo indicado, que la enseñanza de la Historia con foco en la formación ciudadana es posible si el aprendizaje se orienta a la problematización del pasado a través de la discusión de cuestiones controversiales que permitan a los y las estudiantes reconocer múltiples perspectivas en la explicación del pasado, así como les permite enfrentarse a el ejercicio de la discusión y deliberación con la comunidad (Barton \& McCully, 2007; Goldberg \& Savenije, 2018; Santisteban, 2019).

La ciudadanía alentada en esta tercera perspectiva es de tipo máxima por cuanto sus objetivos persiguen que los y las estudiantes se formen en la ciudadanía desde una perspectiva máxima, lo que se expone en que los contenidos seleccionados además de considerar el desarrollo de conocimientos procedimentales para el análisis histórico tienen como foco principal el abordaje de cuestiones controversiales. En cuanto a las metodologías además de favorecer la participación en el análisis y construcción del conocimiento histórico a partir de la inclusión del método histórico en aula, desde esta perspectiva se propone el uso de metodologías que promuevan la discusión y deliberación en el aula, es la intención por favorecer este tipo de metodologías y la centralidad que tiene en los contenidos los temas controversiales, lo que llevan a indicar a esta tercera perspectiva como la que tiene como foco la formación ciudadana por sobre las dos anteriores. 


\subsection{La formación del pensamiento histórico para visibilizar otras narrativas históricas y fortalecer la educación democrática}

Como se ha expuesto para la realización de una práctica de enseñanza que permita construir una Historia escolar que favorezca la formación del pensamiento histórico, la visibilidad de otras narrativas históricas y el fortalecimiento de la educación democrática desde una perspectiva máxima de la ciudadanía, requiere primero reconocer la Historia como una narrativa y desde ahí entender que la Historia Escolar ha construido un discurso social sobre el pasado acorde con los grupos de interés dominantes., También requiere entender que en la transmisión de esas narrativas los y las docentes han sido participantes relevantes, esto hace relevante identificar y analizar las narrativas históricas presente en la Historia Escolar. En segundo lugar, hemos indicado que es fundamental que los y las docentes se posicionen como constructores de currículum y desde esa posición identifiquen las distintas ideologías curriculares presente en la enseñanza de la Historia y desde ahí analicen su práctica y vinculen su quehacer en alguna de estas ideologías, desde el análisis expuesto entendemos que el posicionamiento debería orientarse hacia la tercera perspectiva descrita.

A continuación, describimos como nosotros entendemos el acercamiento práctico a la tercera perspectiva, esto lo hacemos exponiendo primero cómo entendemos el posicionamiento desde lo contextual a lo controversial y socialmente relevante, cómo organizar el abordaje del pensamiento histórico y finalmente cómo transitar hacia la construcción de narrativas que consideren a otros y otras en la Historia escolar.

\subsubsection{Problematizar el contenido desde lo contextual y lo controversial}

La enseñanza de la Historia con foco en la formación de la ciudadanía considerar diseñar el aprendizaje teniendo en cuenta la problematización del contenido, lo que significa establecer una relación entre el contenido curricular y el conocimiento cultural contextual. Por el contenido curricular nos referimos a aquel conocimiento que se desprende del currículum prescrito, ya sea de carácter nacional o el generado por la institución educativa. En cuanto al conocimiento cultural contextual este se refiere a los saberes que surgen del escenario espacial, temporal y cultural en el que se desarrollará enseñanza. Por esto entendemos rescatar los saberes asociados al grupo de estudiantes con los que trabajaremos en el aula, los de la comunidad escolar y los del territorio en el que se inserta el establecimiento educativo. Unido a lo anterior, el conocimiento cultural contextual se refiere también a los que está sucediendo en términos sociales, políticos, culturales y económicos en ese tiempo en particular a nivel local y global.

La problematización emerge de colocar en tensión la relación entre el contenido curricular, el conocimiento ofrecido por el sistema educativo y el conocimiento que existe en el contexto. La problematización dice relación con reflexionar sobre la relevancia social del contenido (Cuesta, 2000). Para plantearse esto algunas preguntas claves a realizarse desde la práctica docente son ¿qué contenidos son relevantes que aprendan mis estudiantes?, ¿por qué y para qué es relevante aprender esos contenidos?, ¿de qué manera estos contenidos curriculares se vinculan con el conocimiento cultural?, ¿cómo el conocimiento cultural contextual se relaciona con el contenido 
curricular? 0 ¿de qué manera el conocimiento cultural contextual hace relevante aprender el contenido curricular?

De la tensión entre contenido curricular y conocimiento cultural contextual surge la problematización que se concreta en una pregunta que orientará el diseño didáctico que llevemos a cabo. De esta manera, la enseñanza tendrá como objeto resolver un problema, resolver una cuestión que es relevante para formar el aprendizaje del conocimiento disciplinar, así como para el contexto. Diversos autores en didáctica de las Ciencias Sociales y de la Historia han vinculado el abordaje del contenido desde esta perspectiva con diversas tradiciones como es el trabajo desde los conceptos sociales claves (Benejam \& Pagès, 2004), el abordaje de problemas socialmente relevantes (Santisteban, 2019; Gutiérrez \& Pagès, 2018) o el abordaje desde lo controversial (Hess, 2009). Nosotros entendemos que más allá del concepto teórico desde el que organicemos la práctica, lo importante es colocar atención en que el contenido a abordar tenga relevancia social para el contexto en el que se desarrollará la docencia.

\subsubsection{Formar el pensamiento histórico y la resignificación del pasado}

La enseñanza de la Historia con foco en la formación ciudadana debe considerar el formar en los y las estudiantes el pensamiento histórico. La importancia de aprender a pensar históricamente desde esta perspectiva se encuentra no sólo en la posibilidad desarrollar una serie de competencias cognitivas disciplinares, sino que es relevante en cuanto permite a las y los estudiantes hacer un análisis crítico del pasado. Esto es importante en cuanto entendemos que la Historia Escolar es una agente importante en la construcción de conciencia histórica, por lo tanto, el aprendizaje de los saberes propios asociados al análisis del pasado permitiría acercarnos al desarrollo de conciencias históricas críticas y/o genéticas en la escuela, cuestión que ha sido relevada como un aporte para la construcción de ciudadanías que avancen en la profundización de la democracia (Pagès, 2009, Santisteban, 2010; Barton \& Levstik, 2009; Seixas, 2017).

Aprender el pensamiento histórico entrega a los y las estudiantes la posibilidad de poder leer y tomar posición sobre el pasado y desde ahí problematizar el presente y proyectar el futuro (Pagès, 2009). Por lo tanto, compartimos con Levesque (2008) la idea sobre que la historia ayuda a una mejor comprensión de la complejidad social y los problemas del presente y que su aprendizaje permitirá a las y los estudiantes convertirse en ciudadanos racionales y críticos. Desde una perspectiva con énfasis en la formación ciudadana se entiende que aprender a pensar históricamente permite que el estudiante resuelva problemas aplicando por una parte sus conocimientos procedimentales de la Historia, pero por sobre todo significa que sea capaz analizar la realidad histórica argumentando sus puntos de vistas y respetando los de los demás, atendiendo a la incorporación de distintos grupos sociales y culturales en la construcción de los procesos históricos y culturales y que sea capaz de proyectar esos saberes a situaciones del mundo actual y del futuro (Pagès, 2009).

Desde la perspectiva que proponemos entendemos que la formación del pensamiento histórico conlleva primero que los y las estudiantes adquieran el conocimiento de una serie de conceptos asociados a la disciplina. Estos conceptos han sido definidos por autores como Levesque (2008) como sustantivos y procedimentales y por Van Boxtel y Van Drie (2018) como 
sustantivos y metaconceptos. Los conceptos sustantivos se refieren al conocimiento histórico asociados a los fenómenos históricos, las estructuras, las personas y los períodos. Mientras que los conceptos procedimentales o metaconceptos están relacionados con los métodos utilizados por los investigadores para describir los procesos y períodos históricos. Limón (2002), por ejemplo, menciona la evidencia, la causa, la explicación, la empatía, el tiempo, el espacio, el cambio, la fuente, el acontecimiento, la descripción y la narración. Ella argumenta que estos metaconceptos son la base del conocimiento histórico y median el entendimiento de los estudiantes de los conceptos sustantivos.

Segundo, para la formación del pensamiento histórico los y las estudiantes deben aprender el pasado planteándose la resolución de un problema, por lo tanto, el estudio del pasado ha de estar orientado desde su inicio por preguntas. La organización del aprendizaje de la Historia a partir de preguntas nos permite acercar a los y las estudiantes a comprender el carácter interpretativo de la Historia (Bain, 2005; Van Boxtel \& Van Drie, 2018).

Tercero, la formación del pensamiento histórico requiere que los y las estudiantes formen el pensamiento temporal para progresivamente avanzar hacia el análisis temporal histórico. Lo anterior, significa que durante los primeros años se debe incentivar a niños y niñas a construir narrativas temporales y a ordenar lo temporal (lenguaje temporal, trabajo sobre la línea de tiempo). Para a continuación avanzar hacia el dominio de conceptos temporales históricos como los de corta duración, mediana duración y larga duración, y el trabajo sobre procedimientos de análisis temporal como cambio y continuidad (Pagès, 2009; Levesque, 2008; Santisteban, 2010; Van Boxtel \& Van Drie, 2018).

Cuarto, formar el pensamiento histórico requiere que los y las estudiantes reconozcan que el acercamiento al pasado es interpretativo y por esto, es fundamental el trabajo con fuentes. Por esto, la enseñanza de la Historia debe comprender que los y las estudiantes logren identificar el rol de las fuentes históricas en la construcción del conocimiento histórico, reconozcan la diversidad de fuentes sobre las cuáles se puede indagar el pasado y es relevante acercar a los y las estudiantes que sean capaces de reconocer el carácter limitado de las fuentes oficiales y las posibilidades que entregan otras fuentes históricas. Los y las estudiantes deben formarse en los procedimientos específicos que permiten el analizar una fuente histórica (Wineburg, 2001; Valle, 2011; Bain, 2005).

Quinto, la formación del pensamiento histórico requiere que los y las estudiantes sean capaces de reconocer el pasado como un tiempo distinto al presente por esto es clave que en la clase de Historia se desarrolle la perspectiva del pasado que considera la contextualización y empatía histórica. A través de la contextualización histórica los y las estudiantes pueden reconocer un fenómeno histórico en un tiempo y espacio social; para describirlo, explicarlo, compararlo y/o evaluarlo (Van Drie \& Van Boxtel, 2018). A través de la empatía histórica los y las estudiantes desarrollan la habilidad para ver y juzgar el pasado en sus propios términos para tratar de entender las mentalidades, los marcos de referencia, las creencias, los valores, las intenciones y las acciones de los agentes históricos utilizando una variedad de evidencias históricas (Yilmaz, 2007). El trabajo de la perspectiva permite que los y las estudiantes, por una parte, eviten juzgar el pasado con ojos del presente y permite, como plantean Barton y Levstik, 
(2009) un acercamiento a la comprensión del actuar de las personas en el pasado, lo que puede ser trasladado al presente para poder comprender la acción de las personas en la comunidad y desde ahí tomar una posición frente a las problemáticas del medio que los rodea.

Sexto, formar el pensamiento histórico requiere dar la oportunidad para que los y las estudiantes puedan explicar los procesos históricos. La explicación histórica permite a los y las estudiantes reconocer la complejidad de los fenómenos históricos y sociales, por esto es clave generar experiencias de aprendizaje en que los y las estudiantes puedan reconocer múltiples perspectivas de los fenómenos históricos, esto requiere colocar atención en particular a sobre que sujetos históricos y espacios incorporamos en la información para desarrollar la explicación histórica, por ejemplo explicar la conquista de América desde la óptica de la corona española, los conquistadores y los pueblos originarios americanos. También es importante que los y las estudiantes aprendan a distinguir que los procesos históricos pueden ser explicados por múltiples causas y que esas causas no siempre son excluyentes entre sí. Para esto es importante avanzar en el trabajo del análisis causal de manera que los estudiantes puedan construir cadenas causales y evaluar la incidencia de distintos agentes y procesos sobre un fenómeno histórico (Chapman, 2003).

Y por último, la formación del pensamiento histórico con foco en la formación ciudadana debe considerar atender al estudio de la agencia histórica. La explicación del pasado conlleva identificar las causas que provocaron acontecimientos y procesos históricos, como hemos dicho estos procesos se explican por causas vinculadas a procesos estructurales y a agentes históricos. Desde una enseñanza de la Historia con foco en la ciudadanía es relevante atender al rol de la agencia histórica, ya que es relevante que los y las estudiantes se pregunten sobre cuáles son los personajes o colectivos que influyeron sobre el desarrollo de los fenómenos históricos, sobre cuáles fueron las consecuencias para distintos agentes, que se pregunten sobre a quienes se reconoce como agentes históricos en los relatos históricos que consumen y se pregunten sobre porqué otros no aparecen. Finalmente entendemos que el trabajo sobre la agencia histórica es relevante para la formación ciudadana, ya que permite a los y las estudiantes reconocer que son personas quienes en el pasado han gestado y dirigido los cambios sociales (Seixas \& Peck, 2004).

\subsubsection{La enseñanza y el aprendizaje de otras narrativas históricas no tradicionales en la Historia Escolar}

Una enseñanza de la Historia con foco en la formación ciudadana debe considerar que el análisis crítico del pasado incorpore la posibilidad de que los y las estudiantes puedan reconocer la agencia histórica de todas las personas, esto significa generar experiencias de aprendizaje histórico que se alejen de los relatos tradicionales que se centraban en la figura del héroe o el personaje excepcional que era romantizado y comenzar a trabajar en la escuela el acercamiento a la construcción de narrativas históricas que incorporen a todas y todos esos sujetos y colectivos históricos no tradicionales o que han sido invisibles para la Historia escolar (Villalón \& Pagès, 2013). Entendemos que este ejercicio es clave para una enseñanza de la Historia con foco en la formación ciudadana porque da la oportunidad de que los y las estudiantes puedan reconocerse en los agentes históricos del pasado y desde ahí reconocer su historicidad y propia agencia, así 
como también permite que los y las estudiantes puedan reconocer la diversidad de perspectivas en la construcción de los fenómenos sociales, dos cuestiones claves para la formación ciudadana.

Para desarrollar otras narrativas históricas en la Historia Escolar consideramos que hay que colocar atención a los siguiente:

- Que el contenido tratado permita conocer el impacto de procesos sociales y/o culturales y cuáles fueron sus consecuencias sobre diversos colectivos no tradicionales (descentrar).

- Incorporar narrativas de distintos sujetos/as históricas no tradicionales sobre los procesos sociales e históricos.

- Que el contenido permita establecer relaciones con el presente y desde ahí establecer discusiones con perspectiva histórica.

- Construir experiencias de aprendizaje que permitan a los y las estudiantes comprender que la Historia es una narrativa. Entender el sentido interpretativo de la Historia.

- Que los y las estudiantes puedan reelaborar el pasado y construir narrativas propias y sobre sujetos/as no tradicionales.

- Que las y los estudiantes tengan acceso a fuentes de distintos sujetos y colectivos históricos no tradicionales y así puedan distinguir las perspectivas de distintos actores y colectivos sobre los hechos del pasado.

- Discutir sobre cómo los significados que tenemos en el presente sobre los procesos históricos están relacionados con la influencia de grupos de interés.

- Analizar de qué manera las narrativas del pasado han sido utilizadas por distintos grupos y esto tiene consecuencias sobre sujetos/as y colectivos históricos.

- Aplicar el conocimiento histórico para la generación de respuestas desde un posicionamiento ético para la resolución de conflictos presente y futuro considerando una perspectiva intercultural/social, es necesario continuar buscando un concepto.

\section{Referencias bibliográficas}

Bain, R. (2005). They thought the world was flat: Applying the principles of how people learn in teaching high school history. National Research Council (2005). How students learn: History in the classroom, (pp.179-213). Washington, DC: The National Academies Press.

Barton, K., \& Levstik, L. (2009). Teaching for the Common Good. New York: Routledge.

Barton, K., \& McCully, A. (2007). Teaching controversial issues...where controversial issues really matter. Teaching History, 127, 13-19.

Benejam, P. \& Pagès, J. (2004). Enseñar y Aprender Ciencias Sociales, Geografía e Historia en la Educación Secundaria. Barcelona: Horsori.

Bolívar, A. (2007). Educación para la ciudadanía. Algo más que una asignatura. España: Graó.

Carr, W. (1998). The curriculum in and for a democratic society. Curriculum Studies, 6(3), 323340.

Carretero, M. (2007). Documentos de Identidad. La construcción de la memoria histórica en un mundo global. Buenos Aires: Paidós. 
Chapman, A. (2003). Camels, diamonds and counterfactuals: A model for teaching causal reasoning. Teaching History, (112), 46-53.

Cox, C., Jaramillo, R., \& Reimers, F. (2005). Educar para la ciudadanía y la democracia en las Américas: Una agenda para la acción. Washington: Banco Interamericano de Desarrollo.

Cuesta, R. (2000) “Uso de abuso de la educación histórica" Revista de las ciencias experimentales y sociales, 14, 23-31.

Eisner, E. (1985). The educational imagination: on the design and evaluation of school programs. New York: Macmillan Publishing Co.

Evans, R. W. (1990). Teacher conceptions of history revisited: Ideology, curriculum, and student belief. Theory \& Research in Social Education, 18(2), 101-138.

Gazmuri, R. (2017). Ideologías curriculares en el debate y negociación del currículum chileno de Historia, Geografía y Ciencias Sociales. Estudios pedagógicos, 43(1), 157-169.

Goldberg, T., \& Savenije, G. M. (2018) Teaching Controversial Historical Issues. En S. Metzger, \& L. Harris (Eds.), The Wiley international handbook of history teaching and learning (pp. 503526). New York: Wiley Blackwell.

Gutiérrez, M., \& Pagès, J. (2018). Pensar para intervenir en la solución de las injusticias sociales. Pereira: Universidad Tecnológica de Pereira. Facultad de Ciencias de la Educación.

Heimberg, C. (2010). ¿Cómo puede orientarse la educación para la ciudadanía hacia la libertad, la responsabilidad y la capacidad de discernimiento de las nuevas generaciones? Iber: Didáctica de las ciencias sociales, geografía e historia, (64), 48-57.

Hess, D. (2009). Controversy in the Classroom: The Democratic Power of Discussion. United States: Routledge.

Kerr, D. (2015). Ciudadanía a nivel nacional regional e internacional: una revisión de enfoques, investigaciones y debates. En C. Cox y J. C. Castillo (Eds.), Aprendizaje de la ciudadanía. Contextos, experiencias y resultados (pp. 11-141). Santiago: Ediciones Universidad Católica de Chile.

Lévesque, S. (2008). Thinking historically: Educating students for the twenty-first century. Canadá: University of Toronto Press.

Lévesque, S., \& Clark, P. (2018). Historical thinking: Definitions and educational applications. En S. Metzger, \& L. Harris (Eds.), The Wiley international handbook of history teaching and learning (pp. 119-48). New York: Wiley Blackwell.

Levstik, L. S., \& Barton, K. C. (2015). Doing history: Investigating with children in elementary and middle schools. United States: Routledge.

Limón, M. (2002). Conceptual change in history. In Reconsidering conceptual change: Issues in Theory and Practice (pp. 259-289). Dordrecht: Springer.

Magendzo, A. \& Gazmuri, R. (2018). La educación del ciudadano. Pensar un currículum para la vida democrática. En A. Arratia \& L. Osandón (Coords.) Políticas para el desarrollo del currículum. Reflexiones y propuestas, (247-270). Santiago: UNESCO.

McGrew, S., \& Byrne, V. L. (2020). Who Is behind this? Preparing high school students to evaluate online content. Journal of Research on Technology in Education, 1-19. 
Nordgren, K., \& Johansson, M. (2015). Intercultural historical learning: a conceptual framework, Journal of Curriculum Studies, 47(1), 1-25

Pagès, J. (1994). La didáctica de las ciencias sociales, el currículum y la formación del profesorado. Signos. Teoría y práctica de la educación, 13, 38-51.

Pagès, J. (2003). Ciudadanía y enseñanza de la historia. Reseñas de Enseñanza de la Historia, 1(1), $11-42$.

Pagès, J. (2009) "El desarrollo del pensamiento histórico como requisito para la formación democrática de la ciudadanía”. Reseñas de Enseñanza de la Historia, 7, 69-91.

Pagès, J., \& Santisteban, A. (2010). La educación para la ciudadanía y la enseñanza de las ciencias sociales, la geografía y la historia. Iber: Didáctica de las ciencias sociales, geografía e historia, (64), 8-18.

Pagès, J., \& Villalón, G. (2013). Los niños y las niñas en la Historia y en los textos históricos escolares. Analecta Calasanctiana, 74(109), 29-66.

Pagés, J., Villalón, G. \& Zamorano, A. (2017). Enseñanza de la Historia y Diversidad Étnica: los casos chileno y español. Educação \& Realidade, 42 (1) 161-182. https://doi.org/10.1590/2175623661186

Rüsen, J. (2008). History: Narration, interpretation, orientation (Vol. 5). New York: Berghahn Books.

Santisteban, A. (2010). La formación de competencias de pensamiento histórico. Clio \& asociados, $14,34-56$.

Santisteban, A. (2019). La enseñanza de las Ciencias Sociales a partir de problemas sociales o temas controvertidos: estado de la cuestión y resultados de una investigación. El Futuro del Pasado, 10, 57-79. https://doi.org/10.14516/fdp.2019.010.001.002

Schiro, M. (2007). Curriculum theory: conflicting visions and enduring concerns. Thousand Oaks: Sage Publications.

Seixas, P. (2017). Historical consciousness and historical thinking. In Palgrave handbook of research in historical culture and education (pp. 59-72). London: Palgrave Macmillan.

Seixas, P., \& Peck, C. (2004). Teaching historical thinking. In A. Sears \& I. Wright (Eds.), Challenges and Prospects for Canadian Social Studies (pp. 109-117). Vancouver: Pacific Educational Press.

Shear, S. B., Knowles, R. T., Soden, G. J., \& Castro, A. J. (2015). Manifesting destiny: Re/presentations of indigenous peoples in K-12 US history standards. Theory \& Research in Social Education, 43(1), 68-101.

Toledo, M. I., \& Gazmuri, R. (2009). Obedientes memoriones o reflexivos pensantes: tensiones entre objetivos identitarios y cognitivos en enseñanza de la historia reciente de Chile en $6^{0}$ año de enseñanza básica. Estudios pedagógicos, 35(2), 155-172.

Valle, A. (2011). El uso de las fuentes escritas en la enseñanza de la Historia. Análisis de textos escolares para tercero y cuarto de secundaria. Educación, 20(38), 81-106.

Van Boxtel, C., \& van Drie, J. (2018). Historical Reasoning: Conceptualizations and Educational Applications. En S. Metzger y L. Harris (Eds.), The Wiley international handbook of history teaching and learning (p. 149-176). New York: Wiley Blackwell. 
Villalón, G. \& Pagès, J. (2013). ¿Quién protagoniza y cómo la historia escolar?: La enseñanza de la historia de los otros y las otras en los textos de estudio de Historia de Chile de educación primaria. Clio \& asociados: La historia enseñada, (17), 119-136.

Villalón, G. \& Pagès, J. (2015). La representación de los y las indígenas en la enseñanza de la historia en la educación básica chilena: El caso de los textos de estudio de historia de Chile. Diálogo andino, (47), 27-36.

White, H. (2014). Metahistoria. La imaginación histórica en la Europa del siglo XIX. México: Fondo de Cultura Económico.

Wineburg, S. (2001) Historical Thinking and other unnatural acts. Charting the future of teaching the past. Filadelfia: Temple University Press.

Yilmaz, K. (2007). Historical empathy and its implications for classroom practices in schools. The History Teacher, 40(3), 331-337.

Zachos D., y Michailidou, A., (2014). 'Others' in Textbooks: The Case of Greek Sixth Grade's History Textbook. Theory in Action, 7(3), 1-25. 